\title{
SLoClas: A DATABASE FOR JOINT SOUND LOCALIZATION AND CLASSIFICATION
}

\author{
Xinyuan Qian, Bidisha Sharma, Amine El Abridi and Haizhou Li \\ Department of Electrical and Computer Engineering, \\ National University of Singapore, Singapore
}

\begin{abstract}
In this work, we present the development of a new database, namely Sound Localization and Classification (SLoClas) corpus, for studying and analyzing sound localization and classification. The corpus contains a total of 23.27 hours of data recorded using a 4-channel microphone array. 10 classes of sounds are played over a loudspeaker at 1.5 meters distance from the array by varying the Direction-of-Arrival (DoA) from $1^{\circ}$ to $360^{\circ}$ at an interval of $5^{\circ}$. To facilitate the study of noise robustness, 6 types of outdoor noise are recorded at 4 DoAs, using the same devices. Moreover, we propose a baseline method, namely Sound Localization and Classification Network (SLCnet) and present the experimental results and analysis conducted on the collected SLoClas database. We achieve the accuracy of $95.21 \%$ and $80.01 \%$ for sound localization and classification, respectively. We publicly release this database and the source code for research purpose.
\end{abstract}

Index Terms - Sound localization, event classification, sound localization and classification database

\section{INTRODUCTION}

Sound Localization and Classification (SLC) refers to estimating the spatial location of a sound source and identifying the type of a sound event through a unified framework. A SLC method enables the autonomous robots to determine sound location and detect sound events for navigation and interaction with the surroundings $[1,2]$. Thus, SLC is useful in smart-city and smart-home applications to automatically specify social or human activities, and assist the hearing impaired to visualize and realize sounds $[3,4,5,6]$. Specifically, a SLC method can be divided into two sub-tasks, Direction-of-Arrival Estimation (DOAE) and Sound Event Classification (SEC).

Most traditional DOAE parametric approaches rely on Time Difference-of-Arrival (TDoA) estimation [7, 8], maximization of Steered Response Power (SRP) [9], or acoustic intensity vector analysis [10]. Whereas the SEC methods rely on both low-level and high-level attributes, including spectrotemporal and Mel Frequency Cepstral Coefficient (MFCC)

This research is supported by the Agency for Science, Technology and Research (A*STAR) under its AME Programmatic Funding Scheme (Project No. A18A2b0046) and Science and Engineering Research Council, Agency of Science, Technology and Research, Singapore, through the National Robotics Program under Grant No. 1922500054. features [11] where classification-based frameworks such as Gaussian Mixture Model (GMM) [8], Hidden Markov Model (HMM) [9], and Support Vector Machine (SVM) [12], are mostly adopted.

Learning-based approaches have the advantages of good generalization under different acoustic environments, where they enable a system to learn the mapping between input features and outputs. Recently, deep-learning based approaches have been successfully applied on DOAE $[13,14]$ and SEC [15]. Since in real-world applications, a sound event may transmit from a specific direction, it is reasonable to combine DOAE and SEC with not only estimating the respective associated spatial location, but also detecting the type of sound [16]. Therefore, it is worthy to investigate the influences and potential relationships between them, which leads to the joint modeling of both tasks [17, 18].

A large-scale database is required for developing deep learning-based approaches to solve real-world problems [19, 20]. Several public databases provide resources to explore solutions for SLC. For the DOAE sub-task, the SSLR database [21] was collected by a 4-channel microphone array mounted on top of a Pepper robot's head. The recorded sounds are transmitted from either a loudspeaker or human subjects. The RSL database [22] includes 22.6 hours of data, which was recorded using a 4-channel microphone array with the sound played over a loudspeaker from 72 different DoAs. For the SEC sub-task, the RWCP sound scene database [19] serves as a long standing corpus, which contains a large number of isolated speech and non-speech environmental sounds. To promote the research and development in SLC, a series of DCASE challenges are organized every year, where consequent different databases with varying recording sound sources and acoustic scenarios, are released [16, 23].

Looking into prospects and progress in the field of SLC technology, a new publicly available database with different sound events is of great importance. A fairly designed database with different sound event classes recorded from several DoAs would help us to solve many real-world SLC problems either by signal processing based analysis, formulating new features or by developing neural networks that learn from a large dataset. In this paper, we propose a sufficiently large database, referred to as SLoClas, which differs from the existing databases in terms of sound classes and 
recording set-up. The SLoClas database is recorded by a 4channel microphone array, including 10 environmental sound classes omitting from 72 different DoAs. We consider realistic sound event classes that include sounds corresponding to bells, bottles, buzzer, cymbals, horn, metal, particle, phone, ring and whistle, each having around 100 examples. The proposed SLC database would provide a common platform to carry on research in DOAE and SEC as two independent tasks, along with the unified SLC task. To facilitate the study of noise robustness, 6 types of noise signals are recorded at 4 DoAs. Additionally, we collect the Time Stretched Pulse (TSP) signals played from all the positions of the loudspeaker, which can be used to calculate the impulse response of the environment [24]. Besides, we present a baseline framework on SLC using the proposed SLoClas database that may serve as a reference system for prospective research. We publicly release this database and the source code of the reference SLC system for research purpose ${ }^{1}$.

This paper is organized as follows. In Section 2, we specify the details of the SLoClas database. To provide a comparative analysis for future technologies, we propose a baseline system, where the input acoustic features and the network architecture are discussed in Section 3. The implementation details and the experimental results are provided in Section 4. Finally, we conclude in Section 5.

\section{SLoClas DATABASE}

In this section, we discuss the SLoClas database design and specify the collection details.

\subsection{Database design}

We use RWCP sound scene classification corpus [19] as the source database, which consists of three broad categories of environmental sounds, including crash sounds of wood, plastic and ceramics, sounds occurred when humans operate on things like spray, saw, claps, coins, books, pipes, telephones, toys, etc. The RWCP database consists of 90 sound classes, where each class comprises of around 100 samples. We note that this database was collected in an anechoic room by B\&K 4134 microphone and DAT recorder in $48 \mathrm{kHz}$ 16bit sampling with a SNR level around 40-50 dB.

We use 10 sound classes from the RWCP corpus (each includes around 100 samples) for the SLoClas corpus collection, and achieve a total of 988 samples. We concatenate these 988 sample sound signals by inserting silence in between, along with the TSP signal combined into a single full length audio file to be played from each position of the loudspeaker. The microphone array is positioned at the room center and the speaker is shifted by varying the angle from $1^{\circ}$ to $360^{\circ}$, at $5^{\circ}$ interval from 1.5 metre distance as shown in Figure 1(a). This results in $(988 \times 72) 71,136$ recorded sounds equivalent to 23.27 hours of data.

\footnotetext{
${ }^{1}$ https://bidishasharma.github.io/SLoClass/
}
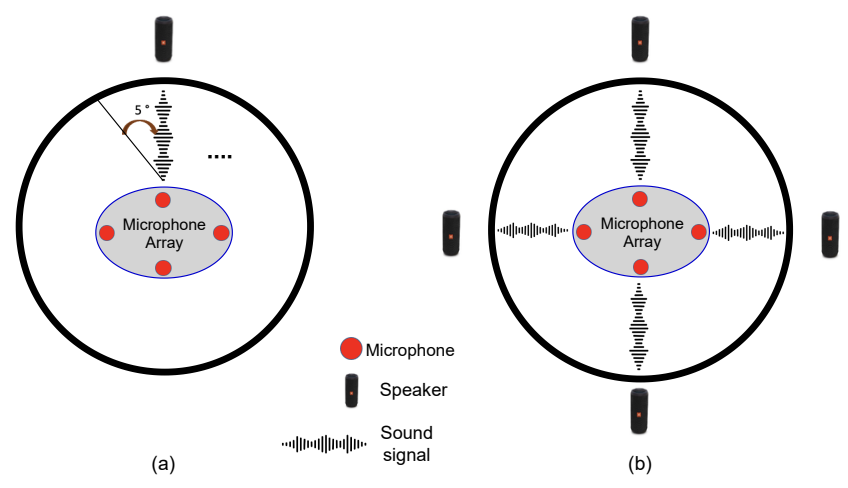

Fig. 1. Visualization of the SLoClas database recording setup when (a) record the sound data, (b) record the noise data.

Besides the aforementioned data, we also record 6 types of outdoor noise (crowd noise, machine noise, traffic and car noise, animal sound, water sound, wind sound) from the PNL 100 Non-speech Sounds database [25]. The noise data was recorded in a similar way at 4 DoAs, keeping the 1.5 metre speaker-array distance as shown in Figure 1(b). We record 64 noise sounds of 6 classes from each DoA, resulting in a total of $(64 \times 4) 256$ noise audio samples.

\subsection{Recording set-up and equipment}

We use a partially reverberation free professional recording studio of dimension $4 \mathrm{~m} \times 5 \mathrm{~m}$. The aforementioned source data is played from a JBL Charge 4 Bluetooth Speaker as discussed in Section 2.1. The loudspeaker volume is kept at half of the highest loudness setting. We use Audacity audio interface [26] connected to the microphone array (ReSpeaker Mic Array v2.0) through a laptop, to capture the audio. The ReSpeaker Mic Array contains 4 high performance digital microphones, with $48 \mathrm{kHz}$ maximum sampling rate. ReSpeaker Mic Array v2.0 supports USB Audio Class 1.0 (UAC 1.0), which is directly connected to the laptop. To avoid any obstruction in the sound propagation path, we mount the microphone array and loudspeaker at the same vertical height using stands and the laptop is operated from ground with minimal noise. We use measuring scale and digital angle finder to mark different DoAs on a 1.5 metre circle on the ground, according to which the loudspeaker location is varied. The marks are at every $5^{\circ}$ azimuth angle ranging from $1^{\circ}$ to $360^{\circ}$. The noise data is recorded using the same equipment setup from $0^{\circ}, 90^{\circ}, 180^{\circ}, 270^{\circ}$ and maintaining the distance of 1.5 meters between the loudspeaker and the microphone array. All audio files are recorded in $f_{s}=48 \mathrm{kHz}$ sampling rate.

\subsection{Post-processing}

For the collected data, we manually annotate the starting point of each full-length recorded audio file with respect to the source audio in Audacity. Using this manually marked starting label, we synchronize the source (loudspeaker) signal and the 4-channel recorded audio signals. Considering 
Table 1. Details of the SLoClas 2021 database in recording set-up, sound data, and noise data (\# indicates number).

\begin{tabular}{|c|c|}
\hline Attribute & Details \\
\hline \multicolumn{2}{|c|}{ 1. Recording set-up: } \\
\hline$f_{s} /$ Bit rate & $48 \mathrm{kHz} / 16$ \\
\hline \multirow[t]{3}{*}{ Sound distance } & $1.5 \mathrm{~m}$ (to the array center) \\
\hline & (a) ReSpeaker Mic Array v2.0 \\
\hline & (b) JBL Bluetooth Speaker \\
\hline \multirow[t]{2}{*}{ Devices } & (c) two 50-cm stainless steel measuring scales \\
\hline & $\begin{array}{l}\text { (d) Digital angle finder }(0.5 \mathrm{~m} / 18 \text {-inch stain- } \\
\text { less steel ruler) }\end{array}$ \\
\hline \multicolumn{2}{|l|}{ 2. Sound data: } \\
\hline \# Sound classes & $\begin{array}{l}10 \text { (bells, bottles, buzzer, cymbals, horn, } \\
\text { metal, particle, phone, ring, whistle) }\end{array}$ \\
\hline \# Samples & 988 per DoA \\
\hline DoA range & $1^{\circ}-360^{\circ}\left(5^{\circ}\right.$ interval $)$ \\
\hline \# DoA & 72 \\
\hline \# Total samples & 71,136 \\
\hline Total duration & 23.27 hours \\
\hline \multicolumn{2}{|l|}{ 3. Noise data: } \\
\hline \# Noise classes & $\begin{array}{l}6 \text { (crowd noise, machine noise, traffic and } \\
\text { car noise, animal sound, water sound, wind } \\
\text { sound) }\end{array}$ \\
\hline \# Samples & 64 per DoA \\
\hline DoA range & $1^{\circ}-360^{\circ}\left(90^{\circ}\right.$ interval $)$ \\
\hline \# DOA & 4 \\
\hline \# Total samples & 256 \\
\hline Total duration & 13 minutes \\
\hline
\end{tabular}

the start of the audio as an anchor point, we segment all the sample sounds with energy based evidence [27, 28, 29] and manual observation. In this way, we achieve 988 segmented audio files and a TSP signal for each DOA angle. We perform a similar processing for the noise data. Details of the proposed SLoClas database are depicted in Table 1.

\section{REFERENCE SYSTEM ON SLOCLAS DATABASE}

In this section, we present a reference system, namely SLCnet, to jointly optimize the SEC and DOAE objectives for sound localization and classification. The network uses the sequential hand-crafted acoustic features (i.e. Generalized Cross Correlation with Phase Transform (GCC-PHAT) and MFCC) as the inputs and produce a DoA label and a sound class label for each audio segment.

\subsection{Features}

\subsubsection{GCC-PHAT}

The GCC-PHAT is widely used to calculate the TDOA between any two microphones in an array $[21,30]$. We adopt it as the audio feature [31] due to its robustness in the noisy and reverberant environment [32] and the fewer tunable parameters than the other counterparts e.g. Short Time Fourier Trans-

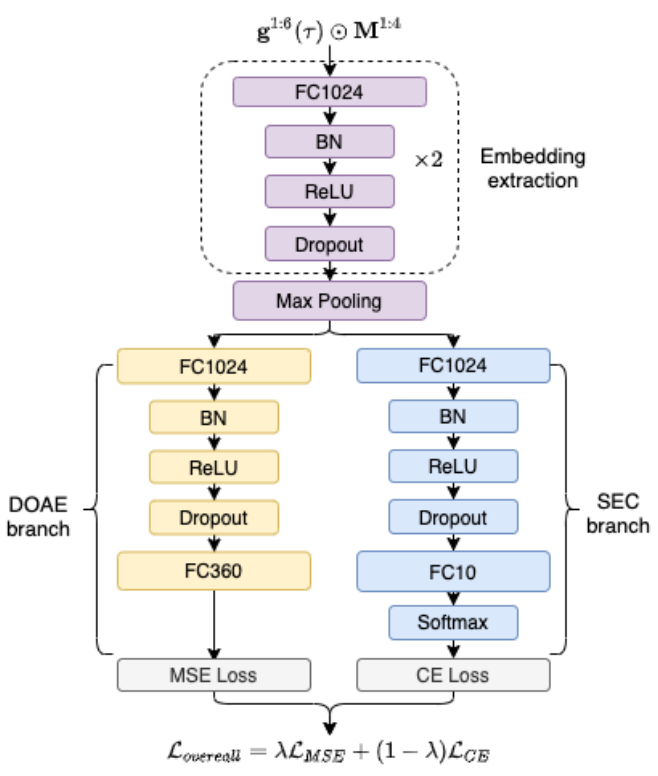

Fig. 2. The proposed SLCnet architecture for SLC, with 3 main parts: (1) the general embedding extraction block (purple), (2) the DOAE branch, (3) the SEC branch. We use the weighted MSE and CE as the final loss. The max pooling is operating along the time dimension (FC: fully-connected layer; BN: batch normalization; $\odot$ indicates concatenation).

form (STFT) [33]. Let $\mathbf{S}_{p_{1}}$ and $\mathbf{S}_{p_{2}}$ be the Fourier transforms of audio sequence at $p_{1}$ and $p_{2}$ channels of the $p$-indexed microphone pair $(p \in[1, \ldots, P])$, respectively. We compute the GCC-PHAT features with different delay lags $\tau$ as:

$$
\mathbf{g}^{p}(\tau)=\sum_{k} \mathcal{R}\left(\frac{\mathbf{S}_{p_{1}}[k]\left(\mathbf{S}_{p_{2}}[k]\right)^{*}}{\left|\mathbf{S}_{p_{1}}[k]\left(\mathbf{S}_{p_{2}}[k]\right)^{*}\right|} e^{j \frac{2 \pi k}{N} \tau}\right)
$$

where, $*$ denotes the complex conjugate operation, $\mathcal{R}$ denotes the real part of complex number and $N$ denotes the FFT length. Here, the delay lag $\tau$ between two signals arrived is reflected in the steering vector $e^{j \frac{2 \pi k}{N} \tau}$ in Eq.1.

\subsection{2. $M F C C$}

The GCC-PHAT feature is not optimal for SEC since it equally sums over all frequency bins while disregarding the sparsity of audio signals in frequency domain where the randomly distributed noise may be stronger than the original audio signal [34]. To explore the frequency domain information, we incorporate the MFCC features from the 4-channel microphone array, and denote the feature as $\mathbf{M}^{m}$ where, $m \in[1, \ldots, M]$ is the microphone index.

\subsection{Network architecture}

The network architecture is shown in Figure 2, which consists of three parts: (1) the embedding extraction (purple), (2) the 


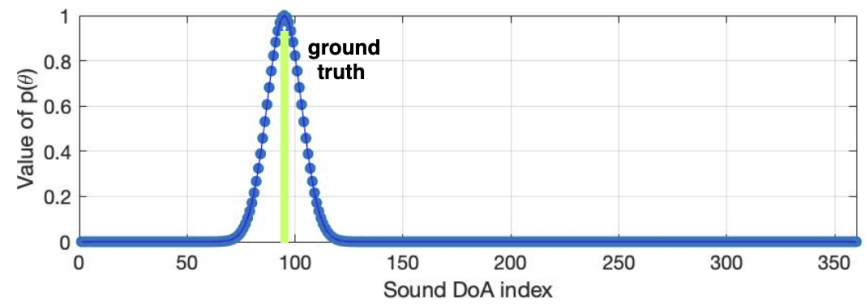

Fig. 3. The sound DoA likelihood-based encoding $p(\theta)$ (Eq.2) that centralized at the ground truth $\dot{\theta}=95^{\circ}$.

SEC branch (blue), and (3) the DOAE block (yellow). Details of each block are described in the next subsections. For simplicity, we eliminate the time index subscript.

\subsubsection{Embedding extraction}

We concatenate the 6-pair GCC-PHAT features and the 4channel MFCC features as the network inputs. We use two stacked Fully Connected (FC) layers, followed by Batch Normalization (BN), ReLU activation, and dropout operation, to generate the latent feature embeddings. A max pooling operation is applied along the time dimension. Then these extracted embeddings are fed into the DOAE and SEC branches.

\subsubsection{DoA estimation}

We use the likelihood-based coding [21] to represent the sound location posterior in each direction. Specifically, each element of the encoded 360-dimensional vector $p(\theta)$ is assigned to a particular azimuth direction $\theta \in\left[1,360^{\circ}\right]$. The value of $p(\theta)$ follows a Gaussian distribution that maximizes at the ground truth DoA:

$$
p(\theta)=\exp \left(-\frac{|\theta-\dot{\theta}|^{2}}{\sigma^{2}}\right)
$$

where, $\dot{\theta}$ is the ground truth, $\sigma$ is a pre-defined constant related to the width of the Gaussian function. For intuitive understanding, in Figure 3 we illustrate an example of $p(\theta)$ (blue dotted curve) centralized at $\dot{\theta}=95^{\circ}$ (vertical green bar).

We predict the sound DoA through a deep-learning network, formulated as:

$$
\hat{p}(\theta)=\mathcal{F}_{\theta}\left(\mathbf{g}^{1: M}, \mathbf{M}^{1: M} \mid \Phi\right)
$$

where, $\Phi$ are the trainable parameters, $\mathcal{F}_{\theta}$ is the proposed DOAE network consists of stacked FC layers, BN, and ReLU activation function. The $\mathcal{F}_{\theta}$ network corresponds to the purple, green and yellow blocks in Figure 2, which outputs the 360-dim DoA posterior.

Finally, we decode the sound DoA estimate by finding the maximum of the output:

$$
\hat{\theta}=\operatorname{argmax}_{\forall \theta} \hat{p}(\theta)
$$

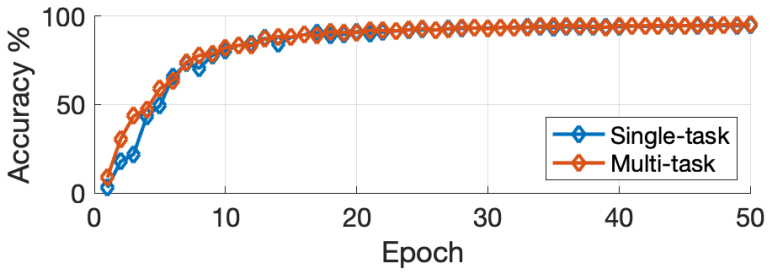

(a) DOAE (sound localization)

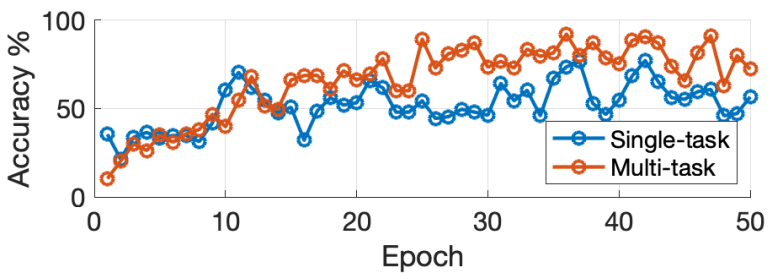

(b) SEC (sound event classification)

Fig. 4. The accuracy (\%) variations on the test set over the training epochs (1) for the DOAE task, and (2) the SEC task.

Instead of CE loss, we adopt the MSE loss to measure the similarity between $p(\theta)$ and $\hat{p}(\theta)$, formulated as:

$$
\mathcal{L}_{M S E}=\|p(\theta)-\hat{p}(\theta)\|_{2}^{2}
$$

where, $\|\cdot\|_{2}$ represents $l-2$ distance.

\subsubsection{Event classification}

We use one-hot encoding for the SEC task:

$$
y(\dot{c})=\mathbf{1}_{c=\dot{c}}
$$

where, $c=1, \ldots, C$ is the event class label with $C$ as the total class number and $\dot{c}$ as the ground truth label.

We predict the event class through the SEC network:

$$
\hat{y}(c)=\mathcal{F}_{e}\left(\mathbf{g}^{1: M}, \mathbf{M}^{1: M} \mid \Psi\right)
$$

where, $\Psi$ are the trainable parameters, $\mathcal{F}_{e}$ consists of stacked FC, BN, ReLU activation function, and a softmax layer is applied as the output layer. The SEC network includes the purple, green and blue blocks in Figure 2, which outputs the probability of each sound event.

A CE loss is computed between the SEC estimate and the ground truth sound class, formulated as:

$$
\mathcal{L}_{C E}=-\log \left(\frac{\exp (y(\dot{c}))}{\sum_{c=1}^{C} \exp (\hat{y}(c))}\right)
$$

where, $\hat{y}(c)$ is computed from Eq.7.

To optimize a multitask-based network, the following objective function is used for SLC:

$$
\mathcal{L}_{\text {overall }}=\lambda \mathcal{L}_{M S E}+(1-\lambda) \mathcal{L}_{C E}
$$


where, $\lambda$ is a pre-defined threshold which indicates the weight of the DOAE loss. Moreover, the network corresponds to the single-task DOAE when $\lambda=1$ and corresponds to the singletask SEC when $\lambda=0$.

\section{EXPERIMENTS}

\subsection{Experimental setup}

The GCC-PHAT is computed for every $170 \mathrm{~ms}$ segments with the delay lag $\tau \in[-25,25]$, resulting in 51 coefficients for each microphone pair. Given 6 pairs (select from any two of the 4 microphones) with each pair contributing 51 GCCPHAT coefficients, we obtain 306 GCC-PHAT coefficients. For MFCC features, we average the 4-channel audio into a single-channel audio. We consider a frame size of $20 \mathrm{~ms}$ with $50 \%$ overlapping. The MFCC feature include 13-dimensional MFCC coefficients, its delta and delta-delta features, resulting into a 39-dimensional feature set. We concatenate the MFCC features of 8 successive frames $(170 \mathrm{~ms})$ to form a 312-dimensional feature set. In this way, we synchronize the frames of GCC-PHAT and MFCC, and concatenate both features to derive a 618-dimensional feature set to develop the proposed SLC network.

We use the Adam optimizer [35]. All models are trained for 50 epochs with a batch size of 32 and a learning rate of 0.001 . Source code in this paper is available ${ }^{2}$.

\subsection{Evaluation metrics}

We evaluate the method performance using the Mean Absolute Error (MAE) and the Accuracy (ACC) metrics.

For DOAE, MAE is computed as the average angular distance between the sound DoA ground truth and estimate:

$$
\operatorname{MAE}\left(^{\circ}\right)=\frac{1}{T} \sum_{t=1}^{T}\left|\dot{\theta}_{t}-\hat{\theta}_{t}\right|
$$

where $\dot{\theta}$ is the DoA ground truth, $\hat{\theta}$ is the estimate from Eq.4, $t$ is an audio segment index, and $T$ is the total segment number.

ACC of DOAE is computed as the percentage of the DoA estimate whose error is smaller than the pre-defined threshold:

$$
\operatorname{ACC}_{\theta}(\%)=\frac{1}{T} \sum_{t=1}^{T} \mathbf{1}_{\left|\hat{\theta}_{t}-\dot{\theta}_{t}\right| \leq \eta}
$$

where, $\mathbf{1}$ is the indicator function, $\eta$ is the DoA error allowance (we set to $5^{\circ}$ in this paper).

For SEC, ACC is also used and computed as:

$$
A C C_{e}(\%)=\frac{1}{T} \sum_{t=1}^{T} \mathbf{1}_{\hat{y}_{t}=\dot{y}_{t}}
$$

Table 2. The experimental results on SLoClas database (NA: result not applicable). " $\downarrow$ " and " $\uparrow "$ indicate the lower the better result and the higher the better result, respectively.

\begin{tabular}{lccc}
\hline \hline & DOAE & SEC & Multi-task \\
\hline MAE $\left(^{\circ}\right) \downarrow$ & $4.68^{\circ}$ & NA & $4.39^{\circ}$ \\
\hline $\mathrm{ACC}_{\theta}(\%) \uparrow$ & $94.93 \%$ & $\mathrm{NA}$ & $95.21 \%$ \\
\hline $\mathrm{ACC}_{e}(\%) \uparrow$ & $\mathrm{NA}$ & $77.23 \%$ & $80.01 \%$ \\
\hline \hline
\end{tabular}

\subsection{Results}

We conduct the experiments on the SLoClas dataset, for which the numerical results are reported in Table 2 in terms of $\mathrm{MAE}, \mathrm{ACC}_{e}, \mathrm{ACC}_{\theta}$, respectively.

For the DOAE task, we achieve the MAE of $4.68^{\circ}$ and the accuracy of $94.93 \%$. For the SEC task, an accuracy of $77.23 \%$ is achieved. For the multi-task training, we report the results with $\lambda=0.99$ (Eq.9) and achieve the MAE of $4.39^{\circ}$, $\mathrm{ACC}_{\theta}$ of $95.21 \%$, and $\mathrm{ACC}_{e}$ of $80.01 \%$. Compared to the individual task, the multi-task training brings benefits to each of the DOAE and SEC branch.

Figure 4 illustrates the variations of the accuracy (\%) on the SLC test set over 50 training epochs where the blue colour corresponds to the single-task training (use a single loss function, either Eq.5 or Eq.8) and the red colour corresponds to the multi-task training. In particular, Figure 4(a) represents the DOAE task and Figure 4(b) represents the SEC task, respectively. From Figure 4(a), we can visualize that with the increasing training epoch, the DOAE performance becomes stable with the resulting $\mathrm{ACC}_{\theta}>90 \%$. Besides, the multitask training strategy doesn't bring significant improvement for DOAE. From Figure 4(b), we can observe a higher performance of the red curve than the blue curve, which indicates the benefits to SEC from mutli-task training, where the final event classification accuracy equals $80 \%$.

\section{CONCLUSION}

In this paper, we present the SLoClas database, to support the study and analysis for joint sound localization and classification. The database contains a total of 23.27 hours of data, including 10 classes of sounds, recorded by a 4-channel microphone array. The sound is played over a loudspeaker from different DoAs, varying from $1^{\circ}$ to $360^{\circ}$ at an interval of $5^{\circ}$. Additionally, to facilitate the study of noise robustness, we record 6 types of outdoor noise at 4 DoAs using the same devices. We also propose a reference system (i.e., SLCnet) with the experiments conducted on SLoClas corpus. We release the source code and the dataset for use in research purpose.

\footnotetext{
${ }^{2}$ https://github.com/catherine-qian/cocosda-SSL. git
} 


\section{REFERENCES}

[1] R. Takeda and K. Komatani, "Discriminative multiple sound source localization based on deep neural networks using independent location model," in 2016 IEEE Spoken Language Technology Workshop (SLT). IEEE, 2016, pp. 603-609.

[2] B. Sharma, R. K. Das, and H. Li, "Multi-level adaptive speech activity detector for speech in naturalistic environments." in INTERSPEECH, 2019, pp. 2015-2019.

[3] J. Wang, X. Qian, Z. Pan, M. Zhang, and H. Li, "GCC-PHAT with speech-oriented attention for robotic sound source localization," 2021, pp. 74-79.

[4] X. Qian, M. Madhavi, Z. Pan, J. Wang, and H. Li, "Multi-target doa estimation with an audio-visual fusion mechanism," 2021, pp. 28142818.

[5] Z. Qiquan, N. Aaron, W. Mingjiang, P. K. Kuldip, and W. Chenxu, "DeepMMSE: A deep learning approach to mmse-based noise power spectral density estimation," pp. 1404-1415, 2021.

[6] R. Tao, Z. Pan, R. Das, X. Qian, M. Z. Shou, and H. Li, "Is someone speaking? exploring long-term temporal features for audio-visual active speaker detection," in ACM Multimedia, 2021, pp. 74-79.

[7] C. Grobler, C. P. Kruger, B. J. Silva, and G. P. Hancke, "Sound based localization and identification in industrial environments," in IECON 2017-43rd Annual Conference of the IEEE Industrial Electronics Society. IEEE, 2017, pp. 6119-6124.

[8] G. Valenzise, L. Gerosa, M. Tagliasacchi, F. Antonacci, and A. Sarti, "Scream and gunshot detection and localization for audio-surveillance systems," in 2007 IEEE Conference on Advanced Video and Signal Based Surveillance. IEEE, 2007, pp. 21-26.

[9] T. Butko, F. G. Pla, C. Segura, C. Nadeu, and J. Hernando, "Two-source acoustic event detection and localization: Online implementation in a smart-room," in 2011 19th European Signal Processing Conference. IEEE, 2011, pp. 1317-1321.

[10] K. Lopatka, J. Kotus, and A. Czyzewski, "Detection, classification and localization of acoustic events in the presence of background noise for acoustic surveillance of hazardous situations," Multimedia Tools and Applications, vol. 75, no. 17, pp. 10 407-10 439, 2016.

[11] P. Foggia, N. Petkov, A. Saggese, N. Strisciuglio, and M. Vento, "Audio surveillance of roads: A system for detecting anomalous sounds," IEEE transactions on intelligent transportation systems, vol. 17, no. 1, pp. 279-288, 2015.

[12] A. Politis and H. Gamper, "Comparing modeled and measurementbased spherical harmonic encoding filters for spherical microphone arrays," in 2017 IEEE Workshop on Applications of Signal Processing to Audio and Acoustics (WASPAA). IEEE, 2017, pp. 224-228.

[13] S. Adavanne, A. Politis, and T. Virtanen, "Direction of arrival estimation for multiple sound sources using convolutional recurrent neural network," in 2018 26th European Signal Processing Conference (EUSIPCO). IEEE, 2018, pp. 1462-1466.

[14] L. Perotin, R. Serizel, E. Vincent, and A. Guerin, "Crnn-based multiple doa estimation using acoustic intensity features for ambisonics recordings," IEEE Journal of Selected Topics in Signal Processing, vol. 13, no. 1, pp. 22-33, 2019.

[15] A. Mesaros, A. Diment, B. Elizalde, T. Heittola, E. Vincent, B. Raj, and T. Virtanen, "Sound event detection in the dcase 2017 challenge," IEEE/ACM Transactions on Audio, Speech, and Language Processing, vol. 27, no. 6, pp. 992-1006, 2019.

[16] Y. Cao, Q. Kong, T. Iqbal, F. An, W. Wang, and M. D. Plumbley, "Polyphonic sound event detection and localization using a two-stage strategy," arXiv preprint arXiv:1905.00268, 2019.

[17] T. Hirvonen, "Classification of spatial audio location and content using convolutional neural networks," in Audio Engineering Society Convention 138. Audio Engineering Society, 2015.
[18] S. Adavanne, A. Politis, J. Nikunen, and T. Virtanen, "Sound event localization and detection of overlapping sources using convolutional recurrent neural networks," IEEE Journal of Selected Topics in Signal Processing, vol. 13, no. 1, pp. 34-48, 2018.

[19] S. Nakamura, K. Hiyane, F. Asano, T. Nishiura, and T. Yamada, "Acoustical sound database in real environments for sound scene understanding and hands-free speech recognition," 2000.

[20] B. Sharma, X. Gao, K. Vijayan, X. Tian, and H. Li, "NHSS: A speech and singing parallel database," Speech Communication, 2021.

[21] W. He, P. Motlicek, and J.-M. Odobez, "Deep neural networks for multiple speaker detection and localization," 2018, pp. 74-79.

[22] R. Sheelvant, B. Sharma, M. Madhavi, R. K. Das, S. Prasanna, and H. Li, "Rsl2019: A realistic speech localization corpus," in 2019 22nd Conference of the Oriental COCOSDA International Committee for the Co-ordination and Standardisation of Speech Databases and Assessment Techniques (O-COCOSDA). IEEE, 2019, pp. 1-6.

[23] A. Politis, S. Adavanne, and T. Virtanen, "A dataset of reverberant spatial sound scenes with moving sources for sound event localization and detection," arXiv preprint arXiv:2006.01919, 2020.

[24] Y. Suzuki, F. Asano, H.-Y. Kim, and T. Sone, "An optimum computergenerated pulse signal suitable for the measurement of very long impulse responses," The Journal of the Acoustical Society of America, vol. 97, no. 2, pp. 1119-1123, 1995.

[25] G. Hu and D. Wang, "A tandem algorithm for pitch estimation and voiced speech segregation," IEEE Transactions on Audio, Speech, and Language Processing, vol. 18, no. 8, pp. 2067-2079, 2010.

[26] “Audacity," https://www.audacityteam.org/.

[27] B. Sharma and S. M. Prasanna, "Vowel onset point detection using sonority information." in INTERSPEECH, 2017, pp. 444-448.

[28] B. D. Sarma, B. Sharma, S. A. Shanmugam, S. M. Prasanna, and H. A. Murthy, "Exploration of vowel onset and offset points for hybrid speech segmentation," in TENCON 2015-2015 IEEE Region 10 Conference. IEEE, 2015, pp. 1-6.

[29] B. Sharma and S. M. Prasanna, "Sonority measurement using system, source, and suprasegmental information," IEEE/ACM Transactions on Audio, Speech, and Language Processing, vol. 25, no. 3, pp. 505-518, 2016.

[30] Z. Pan, M. Zhang, J. Wu, and H. Li, "Multi-tones' phase coding (mtpc) of interaural time difference by spiking neural network," arXiv preprint arXiv:2007.03274, 2020.

[31] C. Knapp and G. Carter, "The generalized correlation method for estimation of time delay," vol. 24, no. 4, pp. 320-327, Aug 1976.

[32] D. Florencio, C. Zhang, and Z. Zhang, "Why does PHAT work well in low noise reverberant environment," Mar 2008, pp. 2565-2568.

[33] S. Chakrabarty and E. A. Habets, "Multi-speaker DoA estimation using deep convolutional networks trained with noise signals," IEEE Journal of Selected Topics in Signal Processing, vol. 13, no. 1, pp. 8-21, Mar 2019.

[34] C. Knapp and G. Carter, "The generalized correlation method for estimation of time delay," vol. 24, no. 4, pp. 320-327, 1976.

[35] D. P. Kingma and J. Ba, "Adam: A method for stochastic optimization," arXiv preprint arXiv:1412.6980, 2014. 